\title{
BMJ Open Patients' and health professionals' attitudes and perceptions towards the initiation of preventive drugs for primary prevention of cardiovascular disease: protocol for a systematic review of qualitative studies
}

\author{
Olla Qadi, Tom Marshall, Nicola Adderley, ${ }^{\circledR}$ Danai Bem
}

To cite: Qadi 0 , Marshall T, Adderley N, et al. Patients' and health professionals' attitudes and perceptions towards the initiation of preventive drugs for primary prevention of cardiovascular disease: protocol for a systematic review of qualitative studies. BMJ Open 2019;9:e025587. doi:10.1136/ bmjopen-2018-025587

- Prepublication history and additional material for this paper are available online. To view these files, please visit the journal online (http://dx.doi. org/10.1136/bmjopen-2018025587).

Received 24 July 2018 Revised 5 March 2019 Accepted 7 March 2019
Check for updates

(C) Author(s) (or their employer(s)) 2019. Re-use permitted under CC BY-NC. No commercial re-use. See rights and permissions. Published by BMJ.

Institute of Applied Health Research, University of Birmingham, Birmingham, West Midlands, UK

Correspondence to

Dr Nicola Adderley;

n.j.adderley@bham.ac.uk

\section{ABSTRACT}

Introduction Lipid-lowering drugs and

antihypertensive agents can be prescribed for the primary prevention of cardiovascular disease. In some cases, patients eligible for primary prevention of cardiovascular disease according to the European guidelines are not always started on preventive drugs. Existing research explores the attitudes of health professionals and patients towards cardiovascular preventive drugs but does not always differentiate between the attitudes towards drug initiation for primary or secondary prevention. We aim to systematically review qualitative studies assessing health professionals' and patients' attitudes and perceptions towards drug initiation for primary prevention of cardiovascular disease.

Methods and analysis MEDLINE, MEDLINE In Process, EMBASE, PsycINF0, CINAHL, Applied Social Sciences Index and Abstracts, Conference Proceedings Citation Index (Web of Science), Healthcare Management Information Consortium, and Open Grey will be searched without restrictions on date or language of publication. Searches will be limited to studies of qualitative design, standalone or in the context of a mixed-method design, focusing on cardiovascular drug initiation for primary prevention. The primary outcome is the attitudes of health professionals and patients towards drug initiation for primary prevention of cardiovascular disease. Two reviewers will independently carry out the study selection, data extraction and quality assessment. The Critical Appraisal Skills Programme Qualitative Research Checklist will be used to assess the quality of included studies. The findings will be analysed using Thomas and Harden's thematic synthesis approach.

Ethics and dissemination This systematic review does not require ethical approval as primary data will not be collected. The results of the study will be published in a peer-reviewed journal and presented at relevant conferences.

PROSPERO registration number CRD42018095346.
Strengths and limitations of this study

- This review will use a systematic approach to summarise qualitative evidence on preventive drug initiation in primary care settings.

- This review will focus on summarising existing evidence regarding drug initiation for primary prevention of cardiovascular disease as recommended by the European guidelines.

- It will provide a better understanding of what influences health professionals' and patients' decisions regarding initiation of preventive treatment.

- The study will not review attitudes towards drug initiation in secondary or tertiary care settings.

- The study will not review studies addressing the initiation of aspirin for the primary prevention of cardiovascular disease.

\section{INTRODUCTION}

Cardiovascular disease (CVD) is the leading cause of deaths worldwide. ${ }^{1}$ It accounts for $26 \%$ of deaths in the UK and $31 \%$ of deaths globally. ${ }^{12}$ One of the ways to prevent CVD is through prescribing drugs for primary prevention. National and international guidelines recommend primary preventive treatment for patients at an increased risk of developing a cardiovascular event. ${ }^{3-6}$ Patients considered at an increased risk include patients with a clinically measured blood pressure of $\geq 140 / 90 \mathrm{~mm} \mathrm{Hg}$ or patients who have a 10 -year CVD risk of $10 \%$ or more. ${ }^{46}$ A patient's risk of developing CVD within the next 10 years can be predicted using a risk assessment tool such as QRISK2. The QRISK2 assessment tool calculates an individual's CVD risk taking into account factors such as age, ethnicity, smoking status, systolic blood pressure, cholesterol/high-density lipoprotein 
(HDL) ratio and body mass index. ${ }^{7}$ The recommendations are supported by evidence from clinical trials demonstrating the beneficial effects of lipid-lowering drugs and antihypertensive agents in the primary prevention of CVD ${ }^{8-11}$ However, studies have reported low prescribing rates of preventive drugs. ${ }^{12-15}$ Patients eligible for statins are undertreated ${ }^{14}{ }^{15}$; one study has reported that $50 \%$ of patients with a CVD risk $\geq 20 \%$ were not prescribed statins for primary prevention. ${ }^{15}$ In addition, the detection and treatment of hypertension remains low in parts of the world. ${ }^{16}{ }^{17}$ Forty-nine per cent of adults with hypertension aged 35-84 years were treated in Japan compared with $80 \%$ in the USA. ${ }^{16}$ However, the initiation rate for antihypertensive drugs in younger eligible adults in the USA is suboptimal. ${ }^{18}$ A study that explored antihypertensive drug initiation among young adults with regular access to primary care found that only $34 \%$ of patients aged 18-39 years were started on antihypertensive drugs compared with $44 \%$ of patients aged $40-59$ years. ${ }^{18}$ This variation in drug initiation observed across countries can be due to multiple factors, including health system, health professional and patient factors. The healthcare system can influence the patient's ability to access health services and the affordability of preventive drugs. The suboptimal prescribing patterns may be a result of health professionals' poor adherence to guideline recommendations. A study conducted in German general practices estimated that around $50 \%$ of general practitioners (GPs) did not adhere to the guidelines. ${ }^{19}$ GPs have expressed concerns regarding the evidence the guidelines were based on and whether following the guidelines will allow them to meet their patients' needs. ${ }^{20}{ }^{21}$ Nevertheless, the variation in prescribing patterns indicates that there are patient-related and GP-related barriers to initiating primary preventive treatment. Previous research identified GP-related barriers such as concerns about patient adherence to medication, overmedicalisation of healthy individuals and side effects. ${ }^{22}$ With respect to patient-related barriers, a study reported that patients preferred making lifestyle changes and had concerns about the side effects of taking medication. ${ }^{23}$ In addition, patients' trust in their GP's medical judgement played a role in accepting preventive treatments. ${ }^{23}$

We are interested in studies that explore the attitudes of health professionals and patients towards initiating treatments for the primary prevention of CVD. A scoping search was carried out to identify existing literature and to estimate the volume of studies available on our topic of interest. The majority of published studies address the issue of adherence to medication or prescribing drugs for secondary prevention. ${ }^{24}{ }^{25}$ However, the search retrieved a number of qualitative studies that investigate patient and health professional-related factors influencing drug prescribing for primary prevention. The search retrieved a systematic review published in 2012 that assessed qualitative literature about initiating and adhering to preventive drugs for CVD. The review discussed factors associated with initiating preventive medication and reported that initiation was influenced by the health professional-patient relationship and the organisational structure of the clinical environment. ${ }^{26}$ The authors focused on starting and adhering to preventive medication with no differentiation between primary and secondary prevention. In addition, studies were excluded from the review based on quality assessment. Our review will consider all primary studies addressing our topic of interest regardless of quality to capture all available evidence regarding prescribing cardiovascular drugs for primary prevention. Furthermore, the search retrieved one recently published systematic review that explored patients' attitudes towards taking statins. However, the review did not explore the attitudes of health professionals towards statins and was restricted to studies in the English language. ${ }^{27}$ The authors explored attitudes only towards statin uptake without differentiating between primary and secondary prevention. Both reviews did not explore grey literature. In this review, we aim to explore grey literature databases to maximise the chances of capturing relevant studies.

Our review will add valuable information to the existing knowledge about CVD prevention. The existing reviews either assess the initiation of a specific drug, such as statins, or focus on the initiation of cardiovascular preventive drugs without differentiating between primary and secondary prevention. In this review, we will include all preventive drugs to provide a comprehensive summary of evidence regarding health professionals' and patients' attitudes towards any cardiovascular drug recommended by the European guidelines for primary prevention. In addition, we chose to focus on drug initiation for primary prevention of CVD because the reasons behind taking cardiovascular preventive drugs such as statins might be different in patients who had a CVD event and patients who are yet to experience a CVD event. The initiation of preventive drugs in a relatively asymptomatic patient can be challenging for both the health professional and the patient, and attitudes relating to this preventive approach need to be identified for successful primary care preventive prescribing. The decision-making process involved in initiating preventive treatments is complex and influenced by multiple factors that relate to both the health professional and the patient. Thus, an up-to-date, methodologically robust systematic review aiming to identify the attitudes and perceptions of health professionals and patients towards the initiation of preventive drugs for the primary prevention of CVD is warranted.

\section{Objectives}

- Explore health professionals' attitudes and perceptions in relation to initiating preventive drugs for primary prevention of CVD in primary care settings.

- Explore patients' attitudes and perceptions towards initiating preventive drugs for primary prevention of CVD in primary care settings. 


\section{METHODS AND ANALYSIS}

This protocol will use the Preferred Reporting Items for Systematic Reviews and Meta-Analyses Protocols (PRISMA-P) guidelines to ensure comprehensive reporting of study items. ${ }^{28}$ The systematic review will follow the reporting guidelines formulated in the Enhancing Transparency in Reporting the Synthesis of Qualitative Research statement. ${ }^{29}$

\section{Information sources and search strategy}

The sample, phenomenon of interest, design, evaluation, research type (SPIDER) tool is considered an alternative to population, intervention, comparison, outcome, study type (PICOS) when addressing a qualitative review question and will be used in the proposed systematic review to formulate the search strategy. ${ }^{30}$ The search strategy will include a combination of free text words and index terms relating to (drug initiation OR prescription OR decision making) and (attitudes OR experiences OR perceptions OR views OR behaviour) and cardiovascular disease. Each element from the SPIDER tool will be included in the search strategy and potential alternative search terms will be included to maximise the chances of retrieving relevant studies. The formulated search strategy will be applied to MEDLINE database (including MEDLINE In Process) then adapted with necessary adjustments for use in other databases. The search strategy for MEDLINE is presented in online supplementary appendix 1 . We will search EMBASE, PsycINFO, CINAHL, and Applied Social Sciences Index and Abstracts for published studies. In addition, the following grey literature sources will be searched: Conference Proceedings Citation Index (Web of Science), Healthcare Management Information Consortium, and Open Grey. The reference lists of included studies will be checked to identify additional eligible studies which were not retrieved by the formulated search strategy. There will be no restriction on date or language of publication. The search will be limited to studies of qualitative design and mixed-methods design with a qualitative component.

\section{Eligibility criteria \\ Sample}

We will include studies of primary care health professionals (GPs and nurse practitioners), in any country, who prescribe cardiovascular preventive drugs. In addition, we will include studies that target patients who are offered a prescription for statins or antihypertensive drugs in a primary care setting. However, studies that specifically focus on drug initiation in older patients will not be included as the considerations for primary prevention of CVD in an older age group are different with additional factors that complicate drug prescription, such as multimorbidity and polypharmacy. Studies that focus on practitioners or patients involved in the process of decision-making or initiation of cardiovascular drugs will be included. Any study that examines practitioners who prescribe preventive drugs and patients who receive such
Table 1 Summary of sample, phenomenon of interest, design, evaluation, research type

\begin{tabular}{|c|c|}
\hline Sample & $\begin{array}{l}\text { Health professionals (general } \\
\text { practitioners or nurse practitioners) } \\
\text { who prescribe statins or } \\
\text { antihypertensive drugs. } \\
\text { Patients eligible for cardiovascular } \\
\text { preventive drugs or offered a } \\
\text { prescription of a statin or an } \\
\text { antihypertensive drug for primary } \\
\text { prevention of cardiovascular disease. }\end{array}$ \\
\hline $\begin{array}{l}\text { Phenomenon of } \\
\text { interest }\end{array}$ & $\begin{array}{l}\text { The initiation or prescription of statins or } \\
\text { antihypertensive drugs. }\end{array}$ \\
\hline Design & $\begin{array}{l}\text { Studies including qualitative data } \\
\text { collection or analysis methods. }\end{array}$ \\
\hline Evaluation & $\begin{array}{l}\text { Attitudes, perceptions, views or } \\
\text { experiences of health professionals } \\
\text { or patients related to the initiation of } \\
\text { cardiovascular preventive drugs. }\end{array}$ \\
\hline Research type & Qualitative and mixed-methods studies. \\
\hline
\end{tabular}

prescriptions for secondary prevention of CVD will be excluded. Studies conducted in secondary care settings will be excluded.

\section{Phenomenon of interest}

Studies will be considered for inclusion if they assess patient or practitioner factors associated with the initiation of cardiovascular preventive drugs in primary care settings. Initiation refers to the prescription of preventive drugs by the practitioner and the patient agreeing to take medication for preventive purposes. Therefore, studies that focus on decision-making or discuss barriers and facilitators to the prescription for primary prevention of CVD will be included. We will exclude studies that focus on adherence and continuation of cardiovascular preventive drugs.

\section{Design/Research type}

Our review aims to look at aspects such as attitudes and perceptions. These are best explored through a qualitative approach. Therefore, any qualitative studies, standalone or in the context of a mixed-methods design, focusing on cardiovascular drug prescription for primary prevention will be included. A summary of SPIDER is provided in table 1 .

\section{Evaluation}

Studies that address the attitudes, perceptions, views or experiences of health professionals or patients involved in the process of cardiovascular preventive drug initiation will be considered for inclusion. To adhere to the European guidelines, we will include studies that target the prescription of statins or antihypertensive drugs. ${ }^{46} \mathrm{We}$ will exclude studies that target the prescription of aspirin as its use for primary prevention is not recommended by several guidelines. ${ }^{51}$ In addition, studies that assess 
the attitudes and perceptions of practitioners or patients towards the prescribing of fibrates, niacin, bile acid sequestrants and omega-3 fatty acid compounds will be excluded as these drugs are not recommended for the primary prevention of CVD. ${ }^{45}$ In some countries, a polypill that contains a lipid-lowering agent and a blood pressure lowering agent is prescribed for CVD risk reduction. ${ }^{32}$ Thus, we will consider studies that assess health professionals' and patients' attitudes towards polypills.

\section{Selection process}

The literature search results will be imported into Endnote V.X8 (Thomson Reuters, New York, USA), to ensure efficient management of references and to facilitate the study selection process. The process of selecting studies will be carried out in two stages by two independent reviewers. The reviewers will follow explicit inclusion/exclusion criteria to minimise potential bias and to ensure minimal influence of the reviewers' preconceptions. The inclusion/exclusion form is presented in online supplementary appendix 2 . The first stage of selection will include screening the titles and abstracts of all identified records against the inclusion criteria. If a study addresses our topic but the abstract lacks sufficient information to assess eligibility for inclusion, the full text will be retrieved to make a definitive decision. In the second stage of selection the two reviewers will retrieve the full texts of included studies and assess them for eligibility. Any disagreements during the selection process will be resolved through discussion. If the two reviewers fail to reach an agreement, a third independent reviewer will be involved for an unbiased decision. The reviewers will keep a record for each article that they have assessed and justify their decision for either inclusion or exclusion. The selection process will be piloted on a small number of studies by the main reviewer to ensure the reliability of the inclusion criteria. The selection process will be illustrated using a PRISMA flow diagram. ${ }^{28}$

\section{Data extraction process}

An electronic standardised data extraction form will be developed to ensure adequate and consistent extraction of all required information. The form will be piloted using a small number of studies to ensure reliability and validity and adjusted if necessary. The electronic form will be used to record extracted data on study characteristics, participants' details, theoretical approach, data collection methods, data analysis and findings (online supplementary appendix 3 ). Once extraction is completed by the two reviewers, the forms will be reviewed, and any discrepancies will be resolved through discussion. If the two reviewers fail to reach agreement, a third reviewer will be involved.

\section{Critical appraisal}

Two independent reviewers will appraise the quality of the included studies using the Critical Appraisal Skills Programme Qualitative Research Checklist. ${ }^{33}$ The assessment of quality will be based on the study aims, methodology, study design, sample recruitment, reflexivity, data collection, data analysis, findings, value of research, and ethics. The reviewers will keep a record of the quality assessment for each study with an explanation of their decision. Any disagreements will be resolved by discussion or referral to a third independent reviewer. Studies will not be excluded from the review based on quality.

\section{Data synthesis}

The NVivo V.10 software will be used to analyse qualitative data. We will adopt a method of thematic synthesis defined by Thomas and Harden for synthesising qualitative data in systematic reviews. ${ }^{34}$ Thematic synthesis includes three stages: First, line-by-line examination of studies' findings and assigning codes to each line of text based on the meaning and content. Second, codes are then grouped into a hierarchical structure and organised as descriptive themes. Finally, analytical themes will be generated to provide interpretations that surpass the findings of the primary studies and ultimately answer our review question. The thematic synthesis will be carried out by two independent reviewers. The reviewers will discuss the codes and themes with an advisory team and then agree on the analytical stage of thematic synthesis.

\section{Patient and public involvement}

This protocol was completed without patient or public involvement. There were no funds or time allocated for patient and public involvement. Therefore, patients were not invited to contribute to the development of this protocol. There are no plans to include patients in any stage of this systematic review. However, the findings of the review will be available to healthcare professionals, policy-makers and the public.

\section{DISCUSSION}

The health professional's decision to prescribe a preventive drug and the patient's willingness to start treatment for preventive purposes is a multifactorial process. It is essential to understand this process of decision-making from a qualitative perspective to enable a more effective approach to CVD prevention. This review will summarise the qualitative evidence available on healthcare professionals' and patients' attitudes towards drug initiation. The findings will help us to understand the complex interaction that occurs during the consultation visit between the patient and their health professional and provide evidence to inform healthcare professionals and policy-makers regarding barriers and facilitators to primary care cardiovascular preventive prescribing.

\section{Ethics and dissemination}

This review will use information available from primary studies. We aim to disseminate the findings of our review 
through publication in a peer-reviewed journal and presentation at a relevant conference.

Contributors $O Q$ formulated the research question, performed the scoping search and wrote the first draft. $\mathrm{OQ}$ and $\mathrm{DB}$ refined research question and search strategy. TM, DB and NA reviewed and revised the draft. All authors read and approved the final manuscript.

Funding $0 Q$ is funded by a governmental scholarship, the study is sponsored by the University of Birmingham.

Competing interests None declared.

Patient consent for publication Not required.

Ethics approval Data will not be collected from individuals, therefore, ethical approval is not required.

Provenance and peer review Not commissioned; externally peer reviewed.

Open access This is an open access article distributed in accordance with the Creative Commons Attribution Non Commercial (CC BY-NC 4.0) license, which permits others to distribute, remix, adapt, build upon this work non-commercially, and license their derivative works on different terms, provided the original work is properly cited, appropriate credit is given, any changes made indicated, and the use is non-commercial. See: http://creativecommons.org/licenses/by-nc/4.0/.

\section{REFERENCES}

1. World Health Organization. Cardiovascular diseases: Fact sheets. 2017. http://www.who.int/mediacentre/factsheets/fs317/en/ (Accessed 1 Jun 2018).

2. British Heart Foundation. CVD statistics - BHF UK factsheet: British Heart Foundation. 2018. https://www.bhf.org.uk/research/heartstatistics (Accessed 1 June 2018).

3. Boon N, Boyle R, Bradbury K, et al. Joint British Societies consensus recommendations for the prevention of cardiovascular disease (JBS3). Heart 2014;100 Suppl 2(Suppl 2):ii1-ii67.

4. National Institute for Health and Care Excellence. Lipid modification: cardiovascular risk assessment and the modification of blood lipids for the primary and secondary prevention of cardiovascular disease. London: NICE. 2014. https://www.nice.org.uk/guidance/cg181 (Accessed 1 Apr 2017).

5. Piepoli MF, Hoes AW, Agewall S, et al. European Guidelines on cardiovascular disease prevention in clinical practice. Atherosclerosis 2016;2016:207-74.

6. National Institute for Health and Care Excellence. Hypertension in adults: diagnosis and management. London: NICE. 2016. https:// www.nice.org.uk/guidance/cg127/chapter/1-Guidance\#initiatingand-monitoring-antihypertensive-drug-treatment-including-bloodpressure-targets-2 (Accessed 1 Apr 2018).

7. Hippisley-Cox J, Coupland C, Vinogradova Y, et al. Predicting cardiovascular risk in England and Wales: prospective derivation and validation of QRISK2. BMJ 2008;336:1475-82.

8. Karmali KN, Lloyd-Jones DM, Berendsen MA, et al. Drugs for primary prevention of atherosclerotic cardiovascular disease: An overview of systematic reviews. JAMA Cardiol 2016;1:341-9.

9. Taylor F, Huffman MD, Macedo AF, et al. Statins for the primary prevention of cardiovascular disease. Cochrane Database Syst Rev 2013;104:Cd004816

10. Thavendiranathan $P$, Bagai $A$, Brookhart MA, et al. Primary prevention of cardiovascular diseases with statin therapy: a meta-analysis of randomized controlled trials. Arch Intern Med 2006;166:2307-13.

11. Chou R, Dana T, Blazina l, et al. Statins for prevention of cardiovascular disease in adults: Evidence report and systematic review for the us preventive services task force. JAMA 2016;316:2008-24.

12. Turner GM, Calvert M, Feltham MG, et al. Under-prescribing of prevention drugs and primary prevention of stroke and transient ischaemic attack in UK general practice: A retrospective analysis. PLoS Med 2016;13:e1002169.

13. Fleetcroft R, Schofield P, Ashworth M. Variations in statin prescribing for primary cardiovascular disease prevention: cross-sectional analysis. BMC Health Serv Res 2014;14:414.

14. Wu J, Zhu S, Yao GL, et al. Patient factors influencing the prescribing of lipid lowering drugs for primary prevention of cardiovascular disease in UK general practice: a national retrospective cohort study. PLoS One 2013;8:e67611.

15. Homer K, Boomla K, Hull S, et al. Statin prescribing for primary prevention of cardiovascular disease: a cross-sectional, observational study. Br J Gen Pract 2015;65:e538-e544.

16. Ikeda N, Sapienza D, Guerrero R, et al. Control of hypertension with medication: a comparative analysis of national surveys in 20 countries. Bull World Health Organ 2014;92:10-19.

17. Baker R, Wilson A, Nockels K, et al. Levels of detection of hypertension in primary medical care and interventions to improve detection: a systematic review of the evidence since 2000. BMJ Open 2018;8:e019965.

18. Johnson HM, Thorpe CT, Bartels CM, et al. Antihypertensive medication initiation among young adults with regular primary care use. J Gen Intern Med 2014;29:723-31.

19. Oriol-Zerbe $\mathrm{C}, \mathrm{Abholz} \mathrm{HH}$. Primary prevention of cardiovascular diseases by lipid-lowering treatment in German general practice: results from GPs ignoring guidelines and risk calculators. Eur J Gen Pract 2007;13:27-34.

20. Carlsen B, Glenton C, Pope C. Thou shalt versus thou shalt not: a meta-synthesis of GPs' attitudes to clinical practice guidelines. $\mathrm{Br} J$ Gen Pract 2007;57:971-8.

21. Carlsen B, Norheim OF. "What lies beneath it all?"--an interview study of GPs' attitudes to the use of guidelines. BMC Health Serv Res 2008;8:218-18.

22. Kedward J, Dakin L. A qualitative study of barriers to the use of statins and the implementation of coronary heart disease prevention in primary care. Br J Gen Pract 2003;53:684-9.

23. Gale NK, Greenfield S, Gill P, et al. Patient and general practitioner attitudes to taking medication to prevent cardiovascular disease after receiving detailed information on risks and benefits of treatment: a qualitative study. BMC Fam Pract 2011;12:59.

24. Fung V, Graetz I, Reed M, et al. Patient-reported adherence to statin therapy, barriers to adherence, and perceptions of cardiovascular risk. PLoS One 2018;13:e0191817.

25. Jamison J, Graffy J, Mullis R, et al. Stroke survivors', caregivers' and GPs' attitudes towards a polypill for the secondary prevention of stroke: a qualitative interview study. BMJ Open 2016;6:e010458.

26. Gale N, Marshall T, Bramley G. Starting and staying on preventive medication for cardiovascular disease. Curr Opin Cardiol 2012;27:533-41

27. Ju A, Hanson CS, Banks E, et al. Patient beliefs and attitudes to taking statins: systematic review of qualitative studies. $\mathrm{Br} J \mathrm{Gen}$ Pract 2018;68:e408-e419.

28. Liberati A, Altman DG, Tetzlaff J, et al. The PRISMA statement for reporting systematic reviews and meta-analyses of studies that evaluate healthcare interventions: explanation and elaboration. BMJ 2009;339:b2700.

29. Tong A, Flemming K, Mclnnes E, et al. Enhancing transparency in reporting the synthesis of qualitative research: ENTREQ. BMC Med Res Methodol 2012;12:181-81.

30. Cooke A, Smith D, Booth A. Beyond PICO: the SPIDER tool for qualitative evidence synthesis. Qual Health Res 2012;22:1435-43.

31. Aspirin: not licensed for primary prevention of thrombotic vascular disease. 2009. https://www.gov.uk/drug-safety-update/aspirin-notlicensed-for-primary-prevention-of-thrombotic-vascular-disease.

32. Huffman MD, Xavier D, Perel P. Uses of polypills for cardiovascular disease and evidence to date. Lancet 2017;389:1055-65 https://doi. org/.

33. Critical Appraisal Skills Programme (CASP). CASP (Qualitative) Checklist. 2018. https://casp-uk.net/wp-content/uploads/2018/03/ CASP-Qualitative-Checklist-Download.pdf (Accessed 1 May 2018)

34. Thomas J, Harden A. Methods for the thematic synthesis of qualitative research in systematic reviews. BMC Med Res Methodol 2008;8:45. 